\title{
DNA FINGERPRINTING OF GRAY WOLVES
}

\author{
PATRICIA P. RABENOLD $\bullet$ RESEARCH SCIENTIST \\ MAURICIO DE GORTARI $\bullet$ GRADUATE STUDENT \\ PURDUE UNIVERSITY $\bullet$ WEST LAFAYETTE $\bullet$ IN
}

\section{$\downarrow \quad$ INTRODUCTION}

Plans and proposals for the restoration of gray wolves (Canis lupus) in Yellowstone National Park are being considered. An important aspect that is contemplated within these plans is the monitoring of the genetic structure of the pack(s) which might be reintroduced. This means that the restored animals, which have known and defined genotypes, could be distinguished from other unrelated and genetically different gray wolves that could be found in the Park. It also means that for every offspring observed within the pack(s) its genetic relationship with the translocated animals could be monitored; that is, establishment of parentage could be routinely carried out. Because distinguishing unrelated wolves phenotypically is practically impossible, it is necessary to rely on a technique capable of revealing sufficient genetic variation to allow individual identification of the relocated wolves, and assignment of parents to the offspring produced within the pack(s).

We used the molecular biological technique called DNA fingerprinting (Jeffreys et al. 1985a) to address this aspect of the gray wolf relocation plans. This technique is based on finding particular DNA regions called hypervariable minisatellites (Jeffreys et al. 1985b), that consist of very short nucleotide sequences repeated many times in tandem and show multiallelic variation in the number of repeating units in each allele. DNA fingerprinting involves the extraction of DNA from almost any fresh tissue (blood, skin, semen, etc.), followed by its digestion with an enzyme (restriction endonuclease), that cuts the DNA molecule at specific base sequences. The DNA fragments produced by this enzymatic digestion are then separated by size along an electrical gradient in an agarose gel. A record of the fragment distribution pattern within the gel is made by blotting the DNA onto a nylon membrane (Southern blotting), to which it is permanently bonded. Those fragments among the total that carry a particular nucleotide sequence are identified via hybridization with a radioactively labeled "probe" molecule carrying the complementary nucleotide sequence (in this case, the core sequence of the tandem repetitive units). The positions at which the probe attaches are recorded by exposing X-ray film to the hybridized membrane. The resulting autoradiograph shows dozens of bands that correspond to the many different fragment sizes bearing the core sequence. This is the "fingerprint".

Genetic variation among individuals leads to enormous differences in banding patterns observed. The banding patterns become more and more dissimilar as individuals diverge genetically until few bands are shared, that is, few DNA fragments of a particular size that hybridize with the probe in one individual also hybridize in another individual, properties that make these fingerprints suitable for individual identification (Jeffreys et al. 1985a). Since fingerprints are inherited according to the rules of Mendelian genetics, they can also be used in paternity testing (Jeffreys et al. 1985b). Helminen et al. (1988) found that in most human paternity cases the sequential use of Jeffreys' probes 33.6 and 33.15 (Jeffreys et al. 1985a) with one restriction enzyme 
digestion was sufficient to identify cases of incorrect paternity.

DNA fingerprinting has found widespread application in parental testing and individual identification in animals. Jeffreys and Morton (1987) successfully tested paternity in dogs and cats, and Morton et al. (1987) established definite proof of paternity in a dogpack. Furthermore, Georges et al. (1988), carrying out DNA fingerprinting in domestic animals using four minisatellite probes, found enough polymorphism to allow individual identification. Several papers have also been published discussing the adequacy of this technique in analyzing parentage in bird species (Burke and Bruford 1987; Rabenold et al. 1990; Rabenold et al. 1991; Wetton et al. 1987).

Because fingerprinting loci assort in Mendelian fashion, the relatedness of individuals drawn at random from a population, including inbred animals, may be estimable by the similarity of their banding patterns, after calibration against proportions of bands shared by individuals of known relatedness (Kuhnlein et al. 1990). Early exploration in using fingerprint pattern similarity as an index of relatedness of individuals within populations (Kuhnlein et al. 1990; Lynch 1990), and as an index of available genetic variation and relationships among populations (Wayne et al. 1991; Gilbert et al. 1990) are encouraging.

We investigated the usefulness of DNA fingerprinting to determine parentage and relatedness among gray wolves, and to distinguish wolves from coyotes. Also, we calculated the probability with which an uncle could mistakenly be assigned as the father of the offspring within packs with two enzymeprobe combinations.

\section{- MATERIALS AND METHODS}

\section{SAMPLES}

\section{GRAY WOLVES}

We worked with a total of 22 gray wolf samples. Almost all of them (21) were blood preserved in a lysis buffer solution containing 100 mM Tris, $100 \mathrm{mM}$ EDTA, and 2\% SDS. The other sample was a piece of ear skin taken from a dead animal preserved in a freezer. Sources from which we received the samples, and their relationships were:
Wolf Park, Battleground, Indiana. We obtained samples from 7 animals. All of them were related to one another. Some of them were the product of an inbred mating (brother $\mathrm{x}$ sister), and 1 of them was the product of 2 consecutive inbred matings (parent $x$ daughter first, and brother $x$ sister second).

Jardin Zoologique du Quebec, Quebec, Canada. They sent us samples from 7 animals of $\mathbf{2}$ different genetic lines. Four of them were arctic wolf (Canis lupus hudsonicus) littermates. The other 3 were a father and $\mathbf{2}$ of its offspring from a different gray wolf subspecies (Canis lupus lycaon).

Milwaukee County Zoo, Milwaukee, Wisconsin. We received samples from 3 of their wolfpack, all of which were arctic wolf littermates.

Toronto Metropolitan Zoo, Toronto, Canada. We received samples from 5 of their arctic wolves. Three of them originated from Wildlife Unlimited in Alberta, Canada, 2 of which are littermates from the same group as the third, which had a different mother and possibly a different father. The other 2, 1 of which is believed to be the offspring of the other by a brother $x$ sister mating, had their origin in the Northwest Territories, Canada.

The genetic lines represented at the different zoos were completely unrelated.

\section{COYOTES}

We worked with 6 coyote samples of unknown origin and relatedness. The samples were frozen tongues from another experiment.

\section{DNA EXTRACTIONS, ELECTROPHORESIS, AND SOUTHERN BLOTTING}

Approximately $10 \mathrm{ml}$ of the preserved solution (containing $5 \mathrm{ml}$ of blood and $5 \mathrm{ml}$ of lysis buffer) from the wolves were prepared by incubating at $55^{\circ} \mathrm{C}$ with $2.5 \mathrm{ml}$ of $5 \mathrm{M} \mathrm{NaCl}$ and $100 \mu 1$ of proteinase $\mathrm{K}(10 \mu \mathrm{g} / \mu 1)$. Small pieces $\left(1 \mathrm{~mm}^{2}\right)$ of the wolf skin and the coyote tongues were macerated in liquid nitrogen and incubated at the same temperature in a solution with $300 \mu 1$ of phosphate-buffered saline (PBS) $\left(2.7 \mathrm{mM} \mathrm{KC1}, 8 \mathrm{mM} \mathrm{Na} \mathrm{HPO}_{4}, 1.5 \mathrm{mM}\right.$ $\mathrm{KH}_{2} \mathrm{PO}_{4}, 14 \mathrm{mM} \mathrm{NaCl}, 6 \mathrm{mM}$ EDTA), $48 \mu 1$ of $10 \%$ SDS, and $30 \mu 1$ of proteinase $\mathrm{K}(10 \mu \mathrm{g} / \mu 1)$. Blood samples were then extracted once with an 
equal volume of phenol, once with one-half volume each of phenol and CIA (Chloroform:isoamyl alcohol at 24:1), and a final extraction with an equal volume of CIA. DNA was precipitated once using 0.05 volumes of $5 \mathrm{M} \mathrm{NaC1}$ and 2 volumes of cold ethanol. After air-drying overnight, DNA pellets were rinsed with $70 \%$ ethanol. Finally, DNA pellets, after air-drying overnight again, were diluted in $1 \mathrm{ml}$ of TE (10 mM Tris at pH 8.0, HC1, $1 \mathrm{mM}$ EDTA). Mean total DNA yield for these samples was 143.4 $+/-62.3 \mu \mathrm{g}$. Samples from coyotes and the wolf skin were extracted twice with an equal volume of phenol, twice with one-half volume each of phenol and CIA, and a final extraction with an equal volume of CIA. They were then extensively dialyzed in $\mathrm{TNE}_{2}$ solution $(10 \mathrm{mM}$ Tris, $10 \mathrm{mM} \mathrm{NaC1}, 2 \mathrm{mM}$ EDTA, pH 8.0). Mean total DNA yield for them was $77.14+/-43.7 \mu \mathrm{g}$.

We conducted a preliminary study, in which DNA from 4 Wolf Park and the 3 Wisconsin wolves were run together twice on the same gel, to determine which enzyme produced more resolvable banding patterns. DNA from the first 7 samples was digested with 5X excess HaeIII, and DNA from the second set with $5 \mathrm{X}$ excess HinfI. From this study we determined that Hinfl enzyme produced a greater number of resolvable bands $(14.86+/-1.95)$ then HaeIII $(8.71+/-2.06)$ for gray wolves. Once we determined which enzyme was better, DNA from the 7 Wolf Park and the 3 Wisconsin wolves was run on the same gel, and DNA from the Quebec and Toronto animals was run together on a separate gel for digestion with Hinfl endonuclease. Also, DNA from the same gray wolf individuals in the first gel and DNA from 6 coyotes was run on another gel. For each individual, $3 \mu \mathrm{g}$ of DNA were digested at $37^{\circ} \mathrm{C}$ for 3-4 hours with excess enzyme (more than 5X for Hinfl) and electrophoresed through $21 \mathrm{~cm}$ long $0.8 \%$ agarose gels at $20 \mathrm{~V}$ for 65 hours; under these conditions, all fragments smaller than $1600 \mathrm{bp}$ generally migrated off the gel. Gels were subsequently stained with ethidium bromide, and photographed under UV illumination; DNA was then Southern transferred to nylon membranes (Nytran or Microseparations Inc.) in 10X SSC $(0.15 \mathrm{M} \mathrm{NaC} 1$, $15 \mathrm{mM}$ trisodium citrate, $\overline{\mathrm{pH}} 7.0$, autoclaved) permanently bonded by baking at $80^{\circ} \mathrm{C}$ under vacuum for 2 hours.

\section{HYBRIDIZATIONS}

The preliminary filter was probed with M13 phage DNA (M13mp8) (Vassart et al. 1987). The final filters containing only DNA from gray wolves were probed with the human minisatellite probes 33.15 and 33.6 (Jeffreys et al. 1985a). The final filter containing DNA from gray wolves and coyotes was only probed with Jeffreys' probe 33.15. Both Jeffreys' probes were labeled by primer extension with $\left.{ }^{32} \mathrm{P}\right]$ dCTP to an average specific activity of $10-$ $60 \times 10^{6} \mathrm{cpm}$. Filters were prehybridized in sealed solution (1.5X SSC, $0.1 \%$ SDS, $5 X$ Denhardt's), and $6 \% \mathrm{~W} / \mathrm{V}$ polyethylene glycol (PEG) at $62^{\circ} \mathrm{C}$. Hybridizations proceeded overnight in the same solution and at the same temperature; following hybridization, 4 30-minute washes in 1.5X SSC and $0.1 \% \mathrm{SDS}$ at $62^{\circ} \mathrm{C}$ were performed. Filters were autoradiographed using Kodak X-Omat film for 3-8 days with a single intensifying screen.

M13 probe was prepared with a Pharmacia oligolabeling kit using [ $\left.{ }^{32} \mathrm{P}\right] \mathrm{dCTP}$ and $100 \mu \mathrm{g}$. DNA per labeling reaction to a specific activity of 30-60 x $10^{6} \mathrm{cpm}$. Filters were prehybridized in $6 \mathrm{X} \mathrm{SSC}$, $0.5 \%$ SDS, $5 X$ Denhardt's solution, and $6 \%$ PEG for 4-6 hours at $60^{\circ} \mathrm{C}$ in the same solution. Filters were then washed twice for 15 minutes at room temperature in $2 \mathrm{X} \mathrm{SSC}, 0.1 \%$ SDS, and once in this solution for 15 minutes at $60^{\circ} \mathrm{C}$. Autoradiography was as for 33.15 and 33.6.

\section{ANALYSIS OF AUTORADIOGRAPHS}

For all analyses, comparisons of banding patterns were confined to bands occurring on the same gel. Pairs of lanes were compared to examine the degree of band sharing between individuals. Only bands representing fragments larger than $3 \mathrm{~Kb}$ were used for analysis as below this size limit bands were consistently too numerous to allow adequate discrimination. Because 2-4 family groups were run on each gel, the combinations included first order relatives (parent-offspring, and full siblings), second order relatives (uncle-nephews from Wolf Park), cousins (only for Wolf Park wolves), and unrelated wolves, in which pairwise comparisons were made between animals from the previously mentioned different genetic lines and origins. 
For the dyadic combinations of lanes within gels the number of bands in common and the number exclusive to each lane was determined. The degree of band sharing between individuals was then calculated as a proportion of the total number of bands in lanes 1 and 2 combined that were found in both (Wetton et al. 1987). In some cases, variation between lanes in band intensity made it necessary to exclude from consideration certain bands in each lane. Therefore, if it was not possible to discern a match for a given band in the other lane, this band was not used in the comparison.

All dyadic combinations were scored independently by two observers. The correlation coefficient between observers was 0.84 . The average of the 2 scores was then taken for all pairwise comparisons, and the mean with its $95 \%$ confidence intervals (Table 1), and $95 \%$ confidence intervals for the distribution of these values (Table 2) were obtained for each of the four categories of relationship with the 2 enzyme-probe combinations used. The expected mean bandsharing value for gray wolf first order relatives was calculated from the observed mean bandsharing for unrelated gray wolves with both enzyme-probe combinations using the formulas provided by Georges et al. (1988; method 1 in Tables 3 and 4). Likewise, using the same formulas with the 2 enzyme-probe combinations, the expected mean bandsharing value for unrelated gray wolves was calculated from the observed mean bandsharing value for gray wolf first order relatives (Method 2 in Tables 3 and 4). A comparison, which is discussed later in the section for results, was made between these observed and expected values.

Because one of the most viable gray wolf reintroduction plans might contemplate the translocation of preestablished pack(s), in which most of the animals are first order relatives, we also used the mean bandsharing value for unrelated gray wolves to calculate the overall probability of misassigning an uncle as the father with two enzyme-probe combinations, using the procedure described by Georges et al. (1988) and Rabenold et al. (1990). Also, due to the possibility of another relocation strategy, in which the release of unrelated individuals having different origin might take place, we used the

Table 1. Mean and $95 \%$ confidence intervals for the proportion of bands shared among all possible gray wolf relationships produced with restriction enzyme. HinfI and Jeffreys' probes 33.15 and 33.6.

Hinfl/33.15

Hinfl/33.6

$\begin{array}{lll}\text { Category Mean } & \text { 95\% C.I. } & \text { Mean }\end{array}$

Gray wolf

first order

relatives

0.721

$(0.665,0.777)$

0.716

$(0.689,0.743)$

Gray wolf

second order

relatives ${ }^{\mathbf{a}}$

0.626

$(0.608,0.645)$

0.527

$(0.505,0.549)$

Gray wolf

cousins $^{b}$

0.646

$(0.528,0.763)$

0.667

$(0.621,0.713)$

Unrelateds

0.395

$(0.364,0.427)$

0.432

$(0.416,0.447)$

a,b: The values for these categories were calculated from pairwise comparisons between Wolf Park individuals. 
Table 2. Mean and $95 \%$ confidence intervals for the distribution of the proportion of bands shared among all possible gray wolf relationships produced with restriction enzyme Hinfl and Jeffreys's probes 33.15 and 33.6.

Hinfl/33.15

Hinfl/33.6

\section{Category}

Mean

$95 \%$ C.I.

Mean

95\% C.I.

Gray wolf

first order

relatives

0.721

$(0.516,0.927)$

0.716

$(0.509,0.924)$

Gray wolf

second order

relatives ${ }^{2}$

0.626

$(0.495,0.758)$

0.527

$(0.431,0.623)$

Gray wolf

cousins $^{\mathrm{b}}$

0.646

$(0.450,0.842)$

0.667

$(0.470,0.864)$

Unrelateds

0.395

$(0.253,0.537)$

0.432

$(0.287,0.576)$

a,b: The values for these categories were calculated from pairwise comparisons between Wolf Park individuals.

Table 3. Results from analysis of DNA fingerprints produced with restriction enzyme HinfI and Jeffreys' probe 33.15 for a panel of 22 gray wolves. Calculations were made by two methods. Method 1 consisted of obtaining the estimates and probabilities from the mean proportion of band sharing between unrelated individuals. Method 2 consisted of obtaining estimates and probabilities from the mean proportion of band sharing between first order relatives.

\begin{tabular}{|c|c|c|}
\hline & Method 1 & Method 2 \\
\hline f (\# of bands) & $14.29+/-2.64$ & $14.29+/-2.64$ \\
\hline x (mean prop. bands shared by unrelateds) & 0.395 & 0.485 \\
\hline$q($ mean allele frequency) & 0.22 & 0.282 \\
\hline h (heterozygosity) ${ }^{b}$ & 0.875 & 0.836 \\
\hline s (mean prop. bands shared by first order relatives) ${ }^{c}$ & 0.678 & 0.721 \\
\hline m (expected \# of maternal bands) ${ }^{d}$ & 9.425 & 10.002 \\
\hline p (expected \# of paternal bands) ${ }^{\circ}$ & 4.865 & 4.301 \\
\hline$P_{u}$ (probab. of misassigning uncle as the father) ${ }^{f}$ & 0.151 & 0.245 \\
\hline$P_{u}$ (with both probes) & 0.04 & 0.07 \\
\hline \multicolumn{3}{|c|}{$\begin{array}{l}\text { a: From } x=2 q-q^{2}\left(\text { first method) (Jeffreys et al. 1985b), or from } s=\left(4+5 q-6 q^{2}+q^{3}\right) / 4(2-q) \text { (second method) }\right. \\
\quad(G e o r g e s \text { et al. 1988). } \\
\text { b: } h=2(1-q) /(2-q)(\text { Georges et al. 1988). } \\
\text { c: } s=\left(4+5 q-6 q^{2}+q^{3}\right) / 4(2-q)(\text { Georges et al. 1988). } \\
\text { d: } m=f\left(1+q-q^{2}\right) /(2-q)(\text { Georges et al. 1988). } \\
\text { e: } p=f\left(q^{2}-q\right) /(2-q) \text { (Rabenold et al. 1990). } \\
\text { f: } p_{u}=s^{p}(\text { Rabenold et al. 1990). }\end{array}$} \\
\hline
\end{tabular}



Table 4. Results from analysis of DNA fingerprints produced with restriction enzyme Hinfl and Jeffreys' probe 33.6 for a panel of 22 gray wolves. Calculations were made by two methods. Method 1 consisted of obtaining the estimates and probabilities from the mean proportion of band sharing between unrelated individuals. Method 2 consisted of obtaining estimates and probabilities from the mean proportion of band sharing between first order relatives.

\begin{tabular}{|c|c|c|}
\hline & Method 1 & Method 2 \\
\hline f (\# of bands) & $12.03+/-3.48$ & $12.03+/-3.48$ \\
\hline x (mean prop. bands shared by unrelateds) & 0.432 & 0.474 \\
\hline$q\left(\right.$ mean allele frequency) ${ }^{2}$ & 0.246 & 0.275 \\
\hline h (heterozygosity) & 0.86 & 0.841 \\
\hline s (mean prop. bands shared by first order relatives) ${ }^{c}$ & 0.696 & 0.716 \\
\hline m (expected \# of maternal bands) ${ }^{d}$ & 8.13 & 8.36 \\
\hline p (expected \# of paternal bands) ${ }^{e}$ & 3.9 & 3.67 \\
\hline$p_{u}$ (probab. of misassigning uncle as the father) ${ }^{f}$ & 0.243 & 0.294 \\
\hline $\mathbf{p u}_{u}$ (with both probes) & 0.04 & 0.07 \\
\hline \multicolumn{3}{|c|}{$\begin{array}{l}\text { a: From } x=2 q-q^{2} \text { (first method) (Jeffreys et al. 1985b), or from } s=\left(4+5 q-6 q^{2}+q^{3}\right) / 4(2-q)(\text { second method) } \\
\text { Georges et al. 1988). } \\
\text { b: } h=2(1-q) / 2-q)(\text { Georges et al. 1988). } \\
\text { c: } s=\left(4+5 q-6 q^{2}+q^{3}\right) / 4(2-q)(\text { Georges et al. 1988). } \\
\text { d: } m=f\left(1+q-q^{3}\right) /(2-q)(\text { Georges et al. 1988). } \\
\text { e: } p=f\left(q^{2}-1\right) /(2-q) \text { (Rabenold et al. 1990). } \\
\text { f: } p_{u}=s^{p} \text { (Rabenold et al. 1990). }\end{array}$} \\
\hline
\end{tabular}

bandsharing value for gray wolf first order relatives to calculate the probability of misassigning an uncle as the father with the same enzyme-probe combinations using the same procedure provided by the same authors. Table 3 displays this and the other genetic estimates produced with restriction enzyme Hinfl and Jeffreys' probe 33.15 . Table 4 shows the values for the same genetic estimates produced with restriction enzyme Hinfl and Jeffreys' probe 33.6.

\section{BAND SHARING}

The expected mean bandsharing values for unrelated gray wolves and for gray wolf first order relatives varied from those observed for the same gray wolf relationships with both enzyme-probe combinations used, after their calculation using the formulas provided by Georges et al. (1988) (Table 3 and 4). We can observe that this variation was larger with HinfI/33.15 enzyme-probe combination than with Hinf1/33.6. This variation might have been due to the fact that the animals we used in our analysis were genetically more related within than between each genetic line. We should recall that the individuals we used in our study came from distant origins and even from 2 different gray wolf subspecies. The observed mean bandsharing value calculated for unrelated gray wolves $(0.395)$ might have been artificially deflated because its calculation included individuals from distant origins and 2 different gray wolf subspecies. On the other hand, the observed mean bandsharing calculated for gray wolf first order relatives $(0.721)$ might have been artificially inflated because its calculation included 
individuals within origins and genetic lines and some known instances of inbreeding. This inflated value for gray wolf first order relatives further suggests the presence of inbreeding within each or some of the gray wolf genetic lines we examined, due probably to closed breeding programs practiced in each institution from which we obtained samples.

Distributions of the proportion of bands shared among the 4 categories of gray wolf relatedness and between gray wolves and coyotes obtained with restriction enzyme 1 Hinfl and 33.15 Jeffreys' probe suggest that distributions for gray wolf close relatives and coyotes do not overlap. Nonetheless, there is some overlap between unrelated gray wolves and the coyotes. Distributions of the proportion of bands shared among the four categories of gray wolf relatedness obtained with Hinfl and 33.6 Jeffreys' probe suggest that distributions for gray wolf first order relatives and unrelateds overlap very little.

\section{ASSIGNMENT OF PARENTAGE}

Following is a description of the procedure we used to calculate the values for the different genetic estimates presented in tables 3 and 4. The results produced with Hinfl/33.15 combination (Table 3) are those used in this description. The results obtained with HinfI/33.6 combination (Table 4) were calculated utilizing the same methodology. We used the formulas provided by Georges et al. (1988) and Rabenold et al. (1990).

From the mean proportion of bands shared between unrelated gray wolves, we estimated both the mean population allele frequency and the probability of a fragment being in the heterozygous state, assuming Hardy-Weinberg equilibrium. Jeffreys et al. (1985b) illustrate that the mean population frequency ( $q$ ) can be found from the equation $x=2 q-$ $q^{2}$, where $x$ is the probability that a band present in 1 individual would also be present in a second, unrelated individual, or 0.395 for Hinfl enzyme. Here $q$ is found to be 0.22 . The probability of a fragment being in the heterozygous state can then be calculated as $h=2(1-q) / 2-q)$ (Georges et al. 1988) and equals 0.875 with the same enzyme, reflecting the high degree of variability observed between fingerprints.

With an estimate for the allele frequency $(q)$, it is also possible to derive an expected level of band sharing among various classes of kin. Georges et al. (1988) provide a method for calculating the expected level of band sharing between first order relatives (parent-offspring, and full siblings): $s=(4+5 q-$ $\left.6 q^{2}+q^{3}\right) / 4(2-q)$. Expected band sharing between gray wolf first order relatives is $\mathbf{0 . 6 7 8}$. From the estimate of allele frequency and the mean number of bands present in one individual (f), we used the formula also provided by Georges et al. (1988) to calculate the expected number of maternal (m) bands present in the offspring, which is 9.4. The expected number of paternal ( $p$ ) bands present in the offspring was calculated using the formula provided by Rabenold et al. (1990); this number was 4.9. Finally, we calculated the probability of mistakenly assigning an uncle as the father $\left(p_{u}\right)$ (Rabenold el al. 1990), which equaled 0.151. Assuming the banding patterns observed using the 2 enzyme-probe combinations were independent, we multiplied $P_{u}$ values produced with both enzyme-probe combinations to obtain an overall probability of misassigning an uncle as the father equal to 0.04 .

The same estimates and probabilities were derived using a similar process, but now calculating first $q$ from the formula depicted above for the probability of band sharing between first order relatives $(\mathrm{s}=.721)$, which in this case is .282 for Hinfl. From this value, we then calculated the rest of the estimates: $\mathrm{h}=0.836, \mathrm{x}=0.485, \mathrm{~m}=10.002$, $\mathrm{p}=4.301, \mathrm{p}_{\mathrm{u}}=0.245$, and overall $\mathrm{P}_{\mathrm{u}}=0.07$.

\section{GENETIC MARKERS}

Two fragments were common to all gray wolves examined with HinfI-33.15 enzyme-probe combination, regardless of geographic origin. To confirm the latter finding, we produced DNA fingerprints from the same preliminary film, which we have worked previously with M13 probe, using now Hinfl-33.15 combination. In this case, the fragments shared among wolves on the other film were not so distinct. So, further research using more samples and from different origin is suggested in the search for genetic markers. No species-specific bands were observed in gray wolves from the fingerprints produced with Hinfl-33.6 combination. Wayne et al. (1991) did not find any marker either with the same enzyme-probe combination that we used. Likewise, preliminary results in coyotes showed no species-specific markers with Hinfl/33.15 enzyme-probe combination. 


\section{$\rightarrow \quad$ DISCUSSION}

Typically, a gray wolf pack is originated by a breeding pair (alpha male and alpha female), and it builds in number with the addition of successive generations of pups, which develop a strong social bond to their parents (Allen 1979). The fact that some of the offspring may remain in the pack and breed (Fritts and Mech 1981), and dispersal strategies displayed by gray wolves (Mech 1987), suggest that inbreeding may occur regularly in this species.

Some authors (Shields 1983; Mech 1987) support the theory that because gray wolves are historically inbred, little inbreeding depression, at least in the short term, should occur within the packs. However, Laikre and Ryman (1991) recently presented evidence of inbreeding depression in a captive gray wolf population. Furthermore, Wayne et al. (1991) suggested that kin recognition, which prevents incestuous matings, might be the reason of the Isle Royal wolves' current decline.

In wild gray wolf populations some members of the pack, at a certain physiological state, disperse to seek a mate and enough resources to propagate (Mech 1987). It is then when the possibility of forming new gray wolf breeding pairs, usually between two individuals from different packs, arises. Occasionally, during the breeding season, a disperser might displace an alpha individual from a different pack, contributing genetic variation which might ameliorate the deleterious effects of inbreeding within the pack.

Because of the gray wolf parental-filial social organization, viable gray wolf relocation plans should contemplate the reintroduction of already established wild wolf pack(s). Should more than one wolf pack be relocated, they should represent different genetic lines. Mixing among different wolf packs' animals, and even among other wild wolf populations that might be living near Yellowstone should be allowed to ameliorate the inbreeding depression effects that might cause a decline in otherwise closed inbred gray wolf populations.

In the situation in which a single pre-established wild pack is released, this analysis suggests an overall error rate of 0.07 in mistakenly assigning an uncle as the father using 2 enzyme-probe combinations. We calculated this probability because it constitutes the most difficult case in which parentage can be established for a given gray wolf offspring within a pack.

Because the distributions of the proportion of bands shared between gray wolf first order relatives and unrelateds detected in our DNA fingerprinting analysis with 2 enzyme-probe combinations overlap very little, it should be possible to distinguish between individuals from the relocated pack and an unrelated gray wolf which might be found in Yellowstone National Park. This could be done by carrying out pairwise comparisons between the suspect individual's DNA fingerprints and those from all animals present in the monitored Yellowstone wolfpack. The distribution, and the mean proportion of bandsharing could be obtained. If those values calculated with either enzyme-probe combination we used lay within the $95 \%$ confidence intervals for the distribution and the mean proportion of bands shared by unrelated gray wolves, and below the lower $95 \%$ confidence limit for the distribution and the mean proportion of bands shared by gray wolf close order relatives calculated for the relocated wolves, the suspect could then be considered as unrelated to Yellowstone's gray wolves.

In the case in which 2 or more packs representing different lineages are relocated, a "foreign" gray wolf could be distinguished from individuals within Yellowstone packs by looking at individual-specific DNA fingerprints; that is, if the suspect's DNA fingerprints do not exactly match one banding pattern from the relocated wolves, then one could determine that this animal is not a member of the monitored packs. If an individual is an offspring derived from the Park's gray wolves, but for whatever reason it is considered a suspect, the analysis of bandsharing between this animal and the rest of the individuals from the monitored pack(s) should be undertaken. If this analysis revealed a proportion of bands shared between the suspect and some of the controlled gray wolves to lie within the $95 \%$ confidence intervals of this estimate for gray wolf first order relatives, DNA from the suspect and from those animals who shared a high proportion of bands with it should be run separately in another gel for parentage testing. If each resolvable band present in the suspect is also present in one Yellowstone gray wolf male-female banding pattern combination, then the animal could be regarded as an offspring derived from that male-female pair. If, on the other hand, 
the suspect's banding pattern could not be matched with any Yellowstone gray wolf male-female DNA fingerprint combination, and the proportion of bands shared between it and the Yellowstone wolves lay within the $95 \%$ confidence intervals for this estimate between unrelated gray wolves, the animal could then be regarded as unrelated to the controlled pack(s), because it was not a product from any of those possible male-female mates.

Another possible gray wolf relocation strategy would be the releasing of individuals from well separated and unrelated wolf packs into the Park. In the case in which individuals from well separated and unrelated wolf packs were released into Yellowstone, our parentage analysis revealed an overall error rate of 0.04 in mistakenly assigning an uncle as the father with 2 enzyme-probe combinations. This rate is slightly lower than that calculated from gray wolf close relatives. Because all individuals would be unrelated one with each other in this case, one could not distinguish, based on the distributions of the proportion of bands shared, between the controlled Yellowstone animals and other gray wolves which might be wandering in the Park. Nevertheless, "foreign" gray wolf could be differentiated from individuals within Yellowstone pack(s) by individualspecific DNA fingerprint matching as previously explained. In the situation in which an individual is an offspring derived from the Park's unrelated gray wolves, but for whatever reason it is considered a suspect, the procedure described above for more than one unrelated packs should be undertaken.

Our analysis revealed that DNA fingerprinting is also useful to distinguish gray wolf close relatives from coyotes. Even though we did not produce very clear fingerprints from our coyote samples, the coyotes' distribution of bands shared between them and gray wolf close relatives do no overlap. If already established gray wolf pack(s) were introduced to Yellowstone, then any coyote could be differentiated from a gray wolf based on the distributions of the proportion of bands shared among them, and by individual-specific DNA fingerprint matching. This latter procedure could also be used to distinguish between a coyote and the wolves if the relocation- strategy involves unrelated gray wolf individuals.

\section{$-\quad$ CONCLUSIONS}

If gray wolf restoration in Yellowstone includes the translocation of already established wild gray wolf pack(s), our analysis revealed that DNA fingerprinting, using restriction enzyme HinfI and either Jeffreys' probe 33.15 or 33.6 , was useful, based on proportions of fragments shared, to distinguish between the relocated wolves and "foreign". wolves which might be found in the Park. A suspect individual could be identified by individual DNA fingerprint matching, too. Also, any animal originated from the originally reintroduced wolves but regarded as a suspect, could be identified by examining the proportion of bands shared between it and the controlled gray wolves, together with parentage testing analysis.

If on the other hand, gray wolf relocation contemplates the translocation of individuals from well separated wild gray wolf packs, our analysis revealed that they could be differentiated from a "foreign" wolf by individual-specific DNA fingerprint matching. Paternity testing analysis could also be carried out to identify any animal originated from the originally relocated wolves. In this case, parentage analysis using two different enzyme-probe combinations was found to be slightly more accurate than in the case of the first possible gray wolf reintroduction strategy.

We also found DNA fingerprinting useful in establishing parentage when more than one enzymeprobe combination was used. In our parentage analysis we considerably reduced the probability of misassigning an uncle as the father using 2 enzymeprobe combinations for both of the most probable gray wolf reintroduction strategies. This, combined with the usefulness of the technique in distinguishing suspect individuals from known gray wolves regardless which relocation strategy is chosen, makes DNA fingerprinting an important technique which could be utilized to monitor and control the genetic structure of the gray wolves which might be released in Yellowstone National Park. 


\section{LITERATURE CITED}

Allen, D. 1979. Wolves of Minong: their vital force role in a wild community. 1st. ed. Houghton Mifflin Company. Boston, MA.

Burke, T. and M. W. Bruford. 1987. DNA fingerprinting in birds. Nature 327:149-152.

Fritts, S. H. and L. D. Mech. 1981. Dynamics, movements, and feeding ecology of a newly protected wolf population in northwestern Minnesota. Wildl. Mon. 80:1-79.

Georges, M., A. S. Lequarre, M. Castelli, R. Hanset and G. Vassart. 1988. DNA fingerprinting in domestic animals using four different minisatellite probes. Cytogenetics and Cell Genetics 47:127-131.

Gilbert, D. A., N. Lehman, S. J. O'Brien and R. K. Wayne. 1990. Genetic fingerprinting reflects population differentiation in the California Channel Island fox. Nature 344:764-766.

Helminen, P., C. Ehnholm, M. L. Lokki, A. J. Jeffreys and L. Peltonen. 1988. Application of DNA "fingerprints" to paternity determinations. The Lancet I:574-575.

Jeffreys, A. J. and D. B. Morton. 1987. DNA fingerprints of dogs and cats. Animal Genetics 18:1-15.

Jeffreys, A. J., V. Wilson and S. L. Thein. 1985a. Individual-specific "fingerprints" of human DNA. Nature 316:76-79.

Jeffreys, A. J., V. Wilson and S. L. Thein. 1985b. Hypervariable "minisatellite" regions in human DNA. Nature 314:67-73.

Kuhnlein, U., D. Zadworny, Y. Dawe, R. W. Fairfull and J. S. Gavora. 1990. Assessment of inbreeding by DNA fingerprinting: Development of a calibration curve using defined strains of chickens. Genetics 125:161-165.

Laikrie, L. and N. Ryman. Inbreeding depression in a captive wolf-(Canis lupus) population. Conserv. Biol. 5:33-40.

Lynch, M. 1990. The similarity index and DNA fingerprinting. Molecular Biol. and Evol. 7:478-484.
Mech, L. D. 1987. Age, season, distance, direction and social aspects of wolf dispersal from a Minnesota pack. Pp. 55-74 In: B. D. ChepkoSade and Z. T. Halpin editors. Mammalian dispersal patterns. The effects of social structure on population genetics. University of Chicago Press, Chicago, Illinois, and London, England.

Morton, D. B., R. E. Yaxley, I. Patel, A. J. Jeffreys, S. J. Howes and P. G. Debenham. 1987. Use of DNA fingerprint analysis in identification of the sire. Vet. Rec. 121:593594.

Rabenold, P. P., K. N. Rabenold, W. H. Piper, J. Haydock and S. W. Zack. 1990. Shared paternity revealed by genetic analysis in cooperatively breeding tropical wrens. Nature 348:538-540.

Rabenold, P. P., K. N. Rabenold, W. H. Piper, M. D. Decker and J. Haydock. 1991. Using DNA fingerprinting to assess kinship and genetic structure in avian populations. Proceedings of the International Congress on Systematics and Evol. Biol. (In press).

Shields, W. M. 1983. Genetic considerations in the management of the wolf and other large vertebrates: and alternative view. Pp. 90-92 In: L. N. Carbyn, editor. Wolves in Canada and Alaska: their status, biology and management. Can. Wild. Ser. Rep. Series, 45. Ottawa.

Vassart, G., M. Georges, R. Monsieur, H. Brocas, A. S. Lequarre and D. Christophe. 1987. A sequence in M13 phage detects hypervariable minisatellites in human and animal DNA. Science 253:683-684.

Wayne, R. K., D. A. Gilbert, N. Lehman, K. Hansen, A. Eisenhawer, D. Girman, R. O. Peterson, L. D. Mech, P. J. P. Gogan, U. S. Seal and R. J. Krumenaker. 1991. Conservation genetics of the endangered Isle royal gray wolf. Conserv. Biol. 5:41-51.

Wetton, J. H., R. E. Carter, D. T. Parkin and D. Walters. 1987. Demographic study of a wild house sparrow population by DNA fingerprinting. Nature 327:147-149. 